\title{
FLUXO DE POTÊNCIA ÓTIMO DESCENTRALIZADO UTILIZANDO ALGORITMOS EVOLUTIVOS MULTIOBJETIVO
}

\author{
Elizete de Andrade Amorim* \\ elizete.amorimegmail.com
}

\author{
Rubén Romero ${ }^{\dagger}$ \\ ruben@dee.feis.unesp.br
}

\author{
José R. S. Mantovani ${ }^{\dagger}$ \\ mant@dee.feis. unesp.br \\ *UNIVERSIDADE FEDERAL DE MATO GROSSO DO SUL - UFMS \\ Laboratório de Pesquisa LABSEEL - Departamento de Engenharia Elétrica - Campus de Campo Grande \\ Caixa Postal 549 - CEP 79070-900 - Campo Grande MS \\ †UNIVERSIDADE ESTADUAL PAULISTA "JÚLIO DE MESQUISTA FILHO" \\ Grupo de Pesquisa em Planejamento de Sistemas Elétricos - Departamento de Engenharia Elétrica - UNESP \\ Caixa Postal 031 -CEP 15385-000 - Ilha Solteira SP
}

\section{RESUMO}

Neste trabalho, apresenta-se o desenvolvimento de uma ferramenta computacional para a solução do problema de fluxo de potência ótimo (FPO) descentralizado. Para esta finalidade o problema de FPO é decomposto em várias áreas, definindo-se vários subproblemas regionais de FPO. O FPO é modelado como um problema de otimização não-linear restrito, em que os custos da geração e das perdas de potência ativa são minimizados simultaneamente. Os subproblemas regionais de FPO são resolvidos através de um algoritmo evolutivo multiobjetivo (AEMO) baseado na teoria de Pareto. No AEMO proposto explora-se um mecanismo de preservação de diversidade entre os indivíduos da população para evitar a convergência prematura e soluções ótimas locais. Para extrair a melhor solução compromisso do conjunto ParetoÓtimo propõem-se um mecanismo de decisão baseado na teoria de conjuntos fuzzy. Além disso, um algoritmo de hierarquia cluster é implementado para reduzir a dimensão do conjunto de Pareto. Para validar a eficiência do modelo e da técnica de solução apresentam-se os resultados obtidos com os sistemas teste RTS-96 e IEEE-354.

Artigo submetido em 16/01/2008 (Id.: 00849)

Revisado em 04/06/2008, 26/01/2009

Aceito sob recomendação do Editor Associado Prof. Eduardo N. Asada
PALAVRAS-ChaVe: Algoritmos Evolutivos, Conjuntos fuzzy, Fluxo de Potência Ótimo, Técnicas de Decomposição, Otimização Multiobjetivo.

\section{ABSTRACT}

This work presents the development of a computational tool for decentralized optimal power flow (OPF) solution. For this purpose, the OPF problem is decoupled into areas defining several regional OPF subproblems. The OPF is modeled as a constrained nonlinear optimization problem, nonconvex, in that the active power losses and optimal dispatch of active and reactive power are minimized simultaneously. Regional OPF subproblems are solved by multiobjective evolutionary algorithm based on the Pareto theory. The proposed approach employs a diversity-preserving mechanism to overcome the premature convergence of algorithm and local optimal solutions. Fuzzy set theory is employed to extract the best compromises of the Pareto set. In addition, a hierarchical clustering algorithm is implemented for reducing Pareto set. To validate the efficiency of the model and the proposed solution technique, the results e analyses of the simulations with the RTS-96 e IEEE-354 test systems are presented.

KEYWORDS: Decentralized Optimal Power Flow, Decom- 
position Technical, Evolutionary Algorithm, Fuzzy Set, Multiobjective Optimization.

\section{INTRODUÇÃO}

Com a desregulamentação no setor de energia elétrica os serviços de geração, transmissão e distribuição passaram a ser oferecidos por diferentes empresas em um ambiente competitivo. A idéia de um sistema de energia elétrica (SEE) centralizado e regulamentado foi substituída por um mercado caracterizado pela independência entre os setores de geração, transmissão, distribuição e comercialização de energia elétrica.

No Brasil o mercado elétrico é composto por quatro submercados (04 áreas). Cada área possui suas próprias características físicas e operacionais, tais como, bacia hidrográfica, sistema de transmissão e um custo de operação diferenciado, embora, a operação do sistema seja totalmente centralizada sem permitir aos agentes locais uma maior liberdade para procurar lucros. Com a liberalização do mercado de energia elétrica este aspecto é aceitável, porque o processo de privatização ainda não foi concluído. Mas a tendência é que a médio e longo prazo o setor elétrico brasileiro esteja totalmente privatizado, migrando de um ambiente centralizado e regulamentado para um ambiente descentralizado, possibilitando a competição no âmbito da geração, distribuição e comercialização de energia.

A desregulamentação do setor elétrico afeta sensivelmente a filosofia de operação e o planejamento de sistema de potência. Desta forma, na utilização ótima dos recursos existentes e planejamento da expansão são necessárias ferramentas de otimização robustas, capazes de atender estes novos requisitos, em especial, aqueles que influenciam na qualidade dos serviços oferecidos e nos aspectos econômicos dos novos investimentos que devem ser previstos nos sistemas.

Nesta nova estrutura do setor elétrico, as concessionárias de energia elétrica estão interessadas em resolver os problemas de planejamento e operação relacionados com a sua área sem considerar as áreas adjacentes pertencentes às outras companhias competidoras. Assim, um dos problemas que deve ser reformulado com o surgimento dos mercados de energia competitivos é o FPO (Carpentier (1962), Dommel e Tinney (1968)) que pode englobar tanto o planejamento da operação (médio e curto prazo) como o planejamento da expansão da rede.

Um sistema de potência interconectado de grande porte pode ser decomposto em áreas independentes, de acordo com o número de áreas existentes. A decomposição dos sistemas de potência é realizada com base em diferentes critérios, tais como, regulamentações legislativas, necessidades técnicas operativas e coordenadas geográficas administrativas. Esta decomposição tem por objetivo permitir a solução do problema de FPO multiárea de modo descentralizado. Vários procedimentos de decomposição têm sido propostos na literatura para resolver o problema de FPO descentralizado. Em particular, as técnicas de relaxação lagrangeana (Aguado et al. (1999), Aguado e Quintana (2001)), procedimentos de relaxação lagrangeana aumentada (Kim e Baldick (1997), Baldick et al. (1999)) e técnicas baseadas no desacoplamento de condições de primeira ordem (Nogales et al. (1999), Bakirtzis e Biskas (2003), Biskas et al. (2005)).

Os algoritmos de decomposição dividem o problema de FPO multiárea em um conjunto de subproblemas regionais independentes. Cada subproblema envolve um subconjunto de variáveis de controle locais com o seu próprio conjunto de restrições e funções objetivo. Para coordenar os subproblemas é necessário gerar um problema mestre com variáveis e restrições (restrições de acoplamento) específicas que não são incorporadas na formulação dos subproblemas regionais. As variáveis específicas do problema mestre são as magnitudes e ângulos de tensão nas barras de fronteiras e os fluxos de potência nas linhas de ligação. Neste procedimento, há uma troca de informações bidirecional entre todos os subproblemas regionais e o problema mestre.

Visando atender às necessidades do mercado de energia elétrica reestruturado no presente trabalho apresenta-se uma ferramenta versátil para solução do problema de FPO de sistemas multiárea com as seguintes características:

1. Otimização de uma área local do sistema de potência interconectado multiárea;

2. Habilidade para considerar várias funções objetivo tais como: minimização dos custos das perdas e geração de potência ativa; despacho ótimo de potência reativa que podem ser especificadas ou não pelo usuário;

3. Detecção dos problemas operacionais e físicos de cada área, tais como: perfil de tensão, déficit de potência reativa, perdas nas linhas de transmissão, transformadores sobrecarregados, etc;

4. Habilidade para tratar um elevado número de variáveis de controle discretas e os subproblemas de potência ativa e reativa simultaneamente.

Para atender estes requisitos, inicialmente, o problema de FPO multiárea é decomposto em áreas definindo um conjunto de subproblemas regionais de FPO, isto é, para cada área do sistema de potência interconectado define-se um subproblema de FPO. Esta decomposição é realizada com o objetivo de resolver o subproblema de FPO de uma determinada 
área sem interferir na solução dos subproblemas das áreas vizinhas (áreas que não estão sendo analisadas). Para decompor um sistema de potência interconectado é necessário que cada área tenha seus próprios geradores e cargas, e que um controlador central troque informações com todas as áreas. Além disso, é necessário determinar os intercâmbios de potência específicos (SIP - Scheduled Interchange Power) (Yu e David (1996)) que cada área deve importar ou exportar para as áreas vizinhas.

No modelo matemático do problema de FPO consideramse a minimização da geração de potência ativa, a minimização dos custos das perdas de potência ativa nas linhas de transmissão e o despacho ótimo de potência reativa em cada área. O FPO é formulado como um problema de otimização não-linear de grande porte, não-convexo, com variáveis de controle contínuas e discretas. Para solução deste problema propõe-se um algoritmo evolutivo multiobjetivo baseado na teoria de Pareto (Deb et al. (2000)). Neste algoritmo explorase um mecanismo de preservação da diversidade para evitar a convergência prematura do algoritmo e ótimos locais. Para extrair a melhor solução compromisso do conjunto Paretoótimo propõem-se um mecanismo de decisão baseado na teoria do conjunto fuzzy (Srinivasan et al. (1994), Dhillon et al. (2002)). Além disso, um algoritmo de hierarquia cluster é implementado para reduzir a dimensão do conjunto de Pareto. Para validar a eficiência do modelo e da técnica de solução apresentam-se os resultados obtidos com os sistemas teste RTS-96 (Grigg et al. (1999)) e IEEE-354 (Aguado e Quintana (2001)).

\section{FORMULAÇÃO E DECOMPOSIÇÃO DO PROBLEMA}

O FPO multiárea pode ser representado matematicamente através de um problema geral de otimização com restrições de igualdade e desigualdade como:

$$
\begin{aligned}
& \min f_{k}(\mathbf{x}, \mathbf{u}) \quad k=1, \ldots, N_{o b j} \\
& \text { Sujeito a: } \\
& g_{l}(\mathbf{x}, \mathbf{u})=0 \quad l=1, \ldots, r \\
& h_{m}(\mathbf{x}, \mathbf{u}) \leq 0 \quad m=1, \ldots, s \\
& \mathbf{x}^{\min } \leq \mathbf{x} \leq \mathbf{x}^{\max } \\
& \mathbf{u}_{C}^{\min } \leq \mathbf{u}_{C} \leq \mathbf{u}_{C}^{\max } \\
& \mathbf{u}_{D} \in D
\end{aligned}
$$

Sendo:

$\mathbf{x}=\left(x_{1}, x_{2}, \ldots, x_{n}\right)^{T} \in \Re^{n}:$ Vetor das variáveis de estado;
$\mathbf{u}=\left[\begin{array}{ll}\mathbf{u}_{C} & \mathbf{u}_{D}\end{array}\right]:$ Vetor das variáveis de controle;

$\mathbf{u}_{C}=\left(u_{C_{1}}, u_{C_{2}}, \ldots, u_{C_{m}}\right)^{T} \in \Re^{n}:$ Variáveis de controle contínuas;

$\mathbf{u}_{D}=\left(u_{D_{1}}, u_{D_{2}}, \ldots, u_{D_{m}}\right)^{T} \in \Re^{n}:$ Variáveis de controle discretas;

$N_{o b j}:$ Número de funções objetivo;

$r$ e $s$ : Número de restrições de igualdade e desigualdade, respectivamente;

$f_{k}(\mathbf{x}, \mathbf{u}): \quad k$-ésima função objetivo;

$g_{l}(\mathbf{x}, \mathbf{u})$ : Conjunto de restrições de igualdade;

$h_{m}(\mathbf{x}, \mathbf{u})$ : Conjunto de restrições de desigualdade;

$\mathbf{x}^{\min } \mathbf{e} \mathbf{x}^{\max }$ : Limites inferiores e superiores das variáveis de estado;

$\mathbf{u}_{C}^{\min }$ e $\mathbf{u}_{C}^{\max }$ : Limites inferiores e superiores das variáveis de controle contínuas;

$D$ : Região de factibilidade das variáveis de controle discretas.

O vetor das variáveis de estado ( $\mathrm{x}$ ) é representado pelos ângulos de fase em todas as barras do sistema e magnitudes das tensões nas barras de carga. As variáveis de controle contínuas $\left(\mathbf{u}_{C}\right)$ são representadas pela potência ativa gerada e magnitudes das tensões nas barras de tensão controlada. As variáveis de controle discretas $\left(\mathbf{u}_{D}\right)$ são representadas pelos transformadores com controle automático de taps e os bancos de capacitores/reatores shunts.

Os termos que compõem a função objetivo (1) de um problema de FPO representam o recurso físico (custos de geração, segurança, qualidade e confiabilidade da operação, etc.) que se deseja otimizar e, desta forma, a sua formulação vai depender dos objetivos do estudo. As restrições de igualdade (2) são as equações não-lineares do fluxo de potência correspondente ao balanço de potência ativa e reativa nas barras do sistema. As restrições de desigualdade (3) são limitações impostas a uma variável ou função, e podem ser classificadas como: restrições físicas e operacionais, restrições de segurança e restrições de acoplamento.

Para decompor o problema (1)-(6) em subproblemas regionais de FPO é necessário estabelecer modelos de restrições de acoplamento adequados devido aos intercâmbios de potência entre as áreas do sistema de potência interconectado. Para manter os fluxos de potência entre as linhas de ligação em seus limites é necessário um controle coordenado entre as áreas e o sistema completo. Além disso, é necessário que cada área do sistema de potência interconectado tenha seus 
próprios geradores e cargas e que um controlador central gerencie as informações entre todas as áreas e determine os intercâmbios de potência especificados (SIP) entre as áreas. Entretanto, nenhuma transação bilateral específica entre as áreas e nem a quantidade de fluxo de potência permitida sobre as linhas de ligação é especificada no modelo de FPO proposto.

Seja $A$ o conjunto das áreas interconectadas e $a$ o índice da área sob análise. Assumindo que as funções objetivo assim como as restrições de igualdade e desigualdade são separáveis em relação às áreas, então os subproblemas regionais do problema (1)-(6) são matematicamente formulados como segue:

$$
\begin{aligned}
& \min f_{k}^{a}\left(\mathbf{x}^{a}, \mathbf{u}^{a}\right) \quad k=1, \ldots, N_{o b j} \quad a \in A \\
& \text { Sujeito a: } \\
& g_{l}^{a}\left(\mathbf{x}^{a}, \mathbf{u}^{a}\right)=0 \quad l=1, \ldots, r \quad a \in A \\
& h_{m}^{a}\left(\mathbf{x}^{a}, \mathbf{u}^{a}\right) \leq 0 \quad m=1, \ldots, s \quad a \in A \\
& w_{e s p}^{a}-\sum_{j \in K a} w_{j}=0 \quad a \in A \\
& \mathbf{x}^{\min , a} \leq \mathbf{x}^{a} \leq \mathbf{x}_{\text {max }, a}^{\max , a} \quad a \in A \\
& \mathbf{u}_{C}^{\min , a} \leq \mathbf{u}_{C}^{a} \leq \mathbf{u}_{C}^{\max } \quad a \in A \\
& \mathbf{u}_{D}^{a} \in D
\end{aligned}
$$

Em que:

$w_{e s p}^{a}$ : Valores de intercâmbio líquido de potência especificados (SIP) entre a área $a$ e suas áreas vizinhas;

$w_{j}$ : Fluxo de potências ativa e reativa sobre a linha de ligação $j$ que interliga a área $a$ e uma área vizinha;

$K a$ : Conjunto das linhas de ligação entre a área $a$ e as áreas vizinhas.

A restrição (10) é denominada restrição de acoplamento e contém variáveis globais necessárias para o controle de potências ativa e reativa entre a área $a$ do sistema e as áreas que são suas vizinhas. Esta restrição é adicionada na formulação do problema de FPO (1)-(6) para permitir que cada empresa que compõe o sistema de potência multiárea opere a sua área independentemente, sem afetar as áreas pertencentes às outras empresas vizinhas.

Esta metodologia permite ao operador do sistema simular o problema de FPO multiárea considerando os modelos de operação centralizada e descentralizada como segue:

Operação Centralizada: A restrição de acoplamento (10) é removida do problema (7)-(13) e o sistema de potência mul- tiárea é considerado como um único sistema interligado resultando em um problema de FPO convencional (1)-(6).

Operação Descentralizada: Cada área do sistema de potência multiárea é analisada independentemente através dos subproblemas regionais de FPO (7)-(13). Esta forma de solução do problema de FPO multiárea é descrita através do seguinte algoritmo:

1. Resolver o problema de fluxo de potência (FP) convencional através do Método de Newton (Monticelli (1983)) para determinar as magnitudes de tensão e os ângulos;

2. Calcular os intercâmbios de potências especificados entre as áreas $\left(w_{e s p}^{a}\right)$ do sistema de potência multiáreas e as áreas vizinhas;

3. Selecionar uma área $a \in A$ para análise;

4. Aplicar a técnica de solução escolhida para resolver o subproblema regional de FPO (7)-(13) referente à área selecionada;

5. Obter os valores otimizados das variáveis de controle locais desta área e resolver o problema de FP convencional para o sistema multiárea completo;

6. Calcular os fluxos de potências sobre as linhas de ligação pertencentes à vizinhança da área $a\left(w_{c a l}^{a}=\right.$ $\left.\sum_{j \in K a} w_{j}\right)$;

7. Repita os passos $i v$ e vi até que a restrição de acoplamento (10) seja satisfeita.

Este algoritmo é implementado através de uma versão sequencial e baseado no princípio da observabilidade local (isto é, um modelo simplificado utilizado para representar o funcionamento e impactos das áreas vizinhas). Embora nos passos $i$ e $v$ os cálculos das equações do fluxo de carga convencional sejam realizados para o sistema completo (todas as áreas interconectadas) esses cálculos são rápidos e toda a base de dados necessária está disponível no controlador central.

Os valores dos intercâmbios de potência especificados $\left(w_{e s p}^{a}\right)$ são obtidos através da solução de um problema de fluxo de carga convencional. Por outro lado, os valores destes intercâmbios podem ser determinados através da solução de um problema de FPO centralizado ou através de contratos bilaterais. Na prática estes intercâmbios podem ser definidos pelos agentes de mercado considerando-se questões técnicas relacionadas com a capacidade excedente de geração das áreas, limites do sistema de transmissão e das linhas de interconexão entre áreas e aspectos econômicos. 
No passo $v$ as variáveis de controle das áreas vizinhas que não estão sob análise são fixas. O controle é completamente realizado pelas próprias áreas e nenhum controlador central participa do processo.

Em uma rede interligada é necessário que sejam controlados os intercâmbios de potência ativa entre as várias áreas que compõem o sistema. O intercâmbio líquido de potência ativa de uma área é definido como a soma algébrica dos fluxos nas linhas e nos transformadores que interligam essa área com as demais (as exportações são consideradas positivas e as importações negativas).

A cada área do sistema é associada uma barra de folga (slack), sendo que a barra de folga de uma das áreas funciona também como barra de folga do sistema (em geral é uma barra do tipo $V \theta$ que serve também como referência angular para o sistema). Com exceção da barra de folga do sistema, as injeções de potências ativa e reativa nas barras de folga das demais áreas são ajustadas para manter os intercâmbios líquidos dessas áreas nos valores especificados. É importante destacar que o controle de intercâmbio regula o intercâmbio total de uma área, ou seja, mantém em um valor especificado a soma algébrica dos intercâmbios individuais nas linhas e nos transformadores que interligam a área com o resto do sistema. Se uma ou mais áreas não possuirem barra de folga, então os seus intercâmbios não podem ser controlados durante o processo iterativo. As barras de folga das áreas, com exceção da barra de folga do sistema (barra $V \theta$ ), são classificadas como do tipo $V$ (só as magnitudes das tensões nodais são especificadas), ou seja, as injeções de potência ativa nessas barras deixam de ser especificadas.

\section{OTIMIZAÇÃO MULTIOBJETIVO}

Um problema de otimização multiobjetivo é caracterizado pela otimização simultânea de várias funções objetivo com diferentes soluções ótimas (Goldberg (1989), Deb et al. (2000)). Geralmente, as funções objetivo de um problema de otimização multiobjetivo são não-comensuráveis e conflitantes entre si e por esta razão não existe uma única solução que seja ótima simultaneamente para todos os objetivos e sim um conjunto de soluções compromisso denominado conjunto eficiente ou Pareto-ótimo.

Para um problema de otimização multiobjetivo com $N_{o b j}$ funções objetivo $\left(f_{i}\right)$ para serem minimizadas simultaneamente, a solução $x_{1}$ domina a solução $x_{2}$ se as condições abaixo são satisfeitas:

$$
\begin{aligned}
& \text { 1. } \forall i \in\left\{1,2, \ldots, N_{o b j}\right\}: f_{i}\left(x_{1}\right)<f_{i}\left(x_{2}\right) \\
& \text { 2. } \exists j \in\left\{1,2, \ldots, N_{o b j}\right\}: f_{j}\left(x_{1}\right) \leq f_{j}\left(x_{2}\right)
\end{aligned}
$$

Se qualquer uma das condições acima é violada, a solução $x_{1}$ não domina a solução $x_{2}$. Se a solução $x_{1}$ domina solução $x_{2}$, então $x_{1}$ é denominada solução não dominada. As soluções que são não-dominadas sobre todo o espaço de busca são chamadas soluções Pareto-ótimas e constituem o conjunto Pareto-ótimo ou a fronteira de Pareto-ótima.

\section{TÉCNICA DE SOLUÇÃO PROPOSTA}

Propõe-se para resolver os subproblemas regionais de FPO (7)-(13) um AEMO baseado em um procedimento de ordenação dos pontos candidatos a serem pontos eficientes da população. Este AEMO é conhecido na literatura técnica como Nondominated Sorting Genetic Algorithm (NSGA) (Srinivas e Deb, (1995)). A diferença desta implementação em relação a um algoritmo genético simples está no modo com que o operador de seleção é realizado. Tanto o operador de recombinação quanto o operador de mutação são os usuais dos algoritmos genéticos. Antes do procedimento de seleção ser aplicado, a população é ordenada com base no nível de não-dominância dos indivíduos, isto é, todas as soluções não-dominadas da população corrente recebem valores altos de aptidão. Esta aptidão é a mesma para todos os indivíduos não-dominados, garantindo assim que todos possuam um mesmo potencial reprodutivo. Para manter a diversidade da população as soluções não-dominadas compartilham os seus valores de aptidão segundo suas distâncias Euclidianas. Finalmente, divide-se o valor da aptidão de cada indivíduo pelo contador de nichos que é proporcional ao número de vizinhos ao seu redor. Este procedimento proporciona a coexistência de pontos ótimos múltiplos na população. $\mathrm{O}$ pior valor de aptidão compartilhada na solução da primeira fronteira não-dominada é então armazenado para uso posterior. Depois que o compartilhamento é executado e que as aptidões são modificadas os indivíduos não-dominados são ignorados temporariamente para processar os demais membros da população. O procedimento para determinar novas soluções não-dominadas (segundo nível) é novamente executado, sendo que agora eles recebem um valor de aptidão um pouco menor que o pior valor de aptidão compartilhada no nível anterior. Uma vez mais o procedimento de compartilhamento é executado entre as soluções não-dominadas do segundo nível e as novas aptidões são calculadas como antes. Este processo é continuado até que todos os membros da população tenham um valor de aptidão compartilhado.

\subsection{Considerações gerais}

O problema de FPO (7)-(13) é um problema de otimização não-linear restrito, não-convexo e com variáveis de controle contínuas e discretas. Este problema é um dos mais complexos na área de otimização, pois geralmente a maioria das soluções contidas no espaço de busca do problema é infactí- 


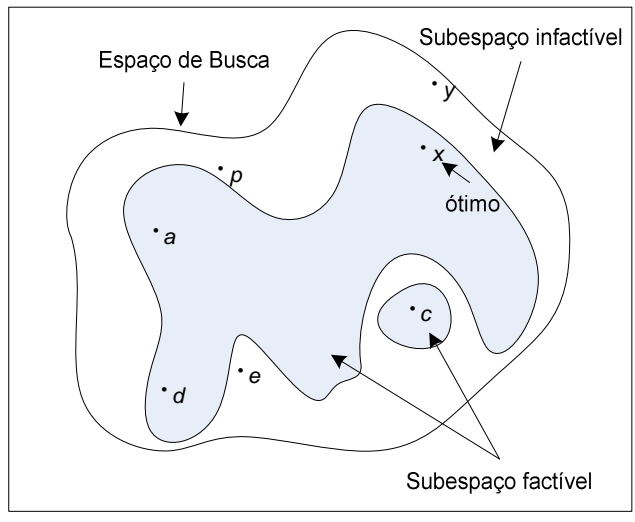

Figura 1: Espaço de busca.

vel. Além disso, os conjuntos de soluções factíveis e infactíveis podem ser não-convexos e não-conectados (ver Figura 1). Estas características causam dificuldades até mesmo para encontrar uma única solução factível.

A maioria dos problemas do mundo real é intratável, ou seja, são problemas para os quais é improvável que se consiga desenvolver métodos analíticos que possam preservar as características físicas e detalhes dos problemas do mundo real. Desta forma, em otimização, a escolha da técnica de solução a ser utilizada para um determinado problema depende, principalmente, da qualidade e precisão da solução que se necessita e da sofisticação do modelo do problema do mundo real.

Os algoritmos evolutivos (AEs) têm sido amplamente utilizados em várias áreas das ciências e engenharias para resolver diversos problemas de otimização. Estes algoritmos podem tratar qualitativamente diferentes tipos de variáveis, tais como: as variáveis discretas, contínuas, reais e inteiras. Além disso, o princípio de não-dominância pode ser facilmente adicionado em seu algoritmo de busca propiciando resolver com êxito os problemas de otimização multiobjetivo (Fonseca e Fleming (1998), Srinivas e Deb (1995), Veldhuizen e Lamont (2000)).

O maior problema quando se aplica os AEs na solução de problemas de otimização restrito é o tratamento das restrições, pois os operadores genéticos utilizados para manipular os cromossomos, frequentemente, geram descendentes infactíveis. Quando as restrições do problema pertencem a intervalos constantes o espaço de busca do problema pode ser considerado como convexo. Entretanto, as restrições nãolineares podem dividir o espaço de busca em vários subespaços não-conectados. Estes subespaços podem ser factíveis e infactíveis e, também, podem ser não-convexos (Figura 1).

Na maioria das técnicas de solução para problemas com res- trições de desigualdade, as restrições violadas são adicionadas à função objetivo do problema através das técnicas de penalidades. Todavia, penalizar as restrições não satisfeitas reduz a aptidão do indivíduo e sua probabilidade de participar do processo de evolução. Por outro lado, é questionável se a penalidade deve impor que todos os indivíduos infactíveis sejam pior que quaisquer outros indivíduos factíveis. Por exemplo, o ponto infactível $y$ na Figura 1 está muito mais próximo do ponto ótimo $x$ que os pontos factíveis $a$, de $c$,consequentemente o ponto $y$ (mesmo sendo infactível) pode fornecer informações mais valiosas se selecionado, entretanto devido à uma política de penalizar em demasia os pontos infactíveis a probabilidade deste ponto ser selecionado para a recombinação e mutação é muito baixa.

Neste trabalho, para contornar estes problemas durante o processamento do AEMO as infactibilidades ocorridas no conjunto de restrições de desigualdade (9) são tratadas como funções objetivo do problema. Esta estratégia permite atender as restrições físicas, operacionais e de acoplamento entre as áreas do sistema, sem comprometer a qualidade das soluções encontradas. Desta forma, os subproblemas de FPO (7)-(13) para cada área $a$ do sistema elétrico de potência multiárea pode ser reescrito como:

\section{A. Funções Objetivo}

\section{A1. Minimização do custo da geração de potência ativa:}

$$
f_{1}=\min \sum_{i \in G} C_{2 i}^{a}\left(P_{g i}^{a}\right)^{2}+C_{1 i}^{a}\left(P_{g i}^{a}\right)+C_{0 i}^{a}, \quad \forall a \in A
$$

Em que:

$P_{g i}^{a}$ : Potência ativa gerada pelo $i$-ésimo gerador da área $a$;

$G$ : Conjunto dos geradores;

$C_{2 i}^{a}, C_{1 i}^{a} \mathbf{e} C_{0 i}^{a}$ : Coeficientes de custo do $i$-ésimo gerador pertencente à área $a$.

\section{A2. Minimização do custo das perdas de potência ativa:}

$$
f_{2}=\min \sum_{j \in N L} C_{P_{J}}^{a} \text { Ploss }_{j}^{a}, \quad \forall a \in A
$$

Em que:

Ploss $_{j}^{a}:$ Perdas nas linhas de transmissão da área $a$; 
$C_{P_{J}}^{a}$ : Coeficientes de custo das perdas na $j$-ésima linha de transmissão da área $a$;

NL: Conjunto das linhas de transmissão.

\section{B. Funções objetivo referentes às restri- ções de desigualdade violadas}

\section{B1. Potência ativa gerada:}

$$
h_{1}=\min \left\{0, P_{g_{\text {ref }}}^{a}-P_{g_{\text {ref }}}^{a, \max }\right\}, \forall a \in A
$$

Sendo:

$P_{g_{r e f}}^{a}$ : Potência ativa gerada na barra slack da área $a$;

$P_{g_{r e f}}^{a, \max }:$ Capacidade máxima de geração de potência ativa na barra slack da área $a$.

\section{B2. Potência reativa gerada:}

$$
h_{2}=\operatorname{Min} \sum_{i \in G} \Delta Q_{g i}^{a}, \quad \forall a \in A
$$

Em que:

$$
\Delta Q_{g i}^{a}=\left\{\begin{array}{cc}
Q_{g i}^{a, \min }-Q_{g i}^{a}, & Q_{g i}^{a}<Q_{g i}^{a, \min } \\
Q_{g i}^{a}-Q_{g i}^{a, \max }, & Q_{g i}^{a}>Q_{g i}^{a, \max } \\
0, & \text { caso contrário }
\end{array}\right.
$$

$Q_{g i}^{a}$ : Potência reativa gerada pelo $i$-ésimo gerador da área $a$

$Q_{g i}^{\min } \mathbf{e} Q_{g i}^{\max }$ : Capacidade mínima e máxima da geração de potência reativa na barra $i$, pertencente à área $a$.

\section{B3. Magnitudes de tensão:}

$$
h_{3}=\operatorname{Min} \sum_{i \in N} \Delta V_{i}^{a}, \forall i \in N, \forall a \in A
$$

Em que:

$$
\Delta V_{i}^{a}=\left\{\begin{array}{cc}
V_{i}^{a, \min }-V_{i}^{a}, \quad V_{i}^{a}<V_{i}^{a, \min } \\
V_{i}^{a}-V_{i}^{a, \max }, \quad V_{i}^{a}>V_{i}^{a, \max } \\
0, & \text { caso contrário }
\end{array}\right.
$$

$N$ : Conjunto das barras do sistema potência;

$V_{i}^{a}$ : Magnitude de tensão na $i$-ésima barra da área $a$;

$V_{i}^{a, \text { min }}$ : Limite inferior da magnitude de tensão na $i$-ésima barra da área $a$;

$V_{i}^{a, \max }:$ Limite superior da magnitude de tensão na $i$-ésima barra da área $a$.

\section{B4. Fluxo de potência nas linhas de transmissão:}

$$
h_{4}=\min \sum_{i, j \in N} \Delta S_{i j}^{a}, \quad \forall a \in A
$$

Sendo:

$$
\Delta S_{i j}^{a}=\left\{\begin{array}{cc}
S_{i j}^{a}-S_{i j}^{a, \max }, & S_{i j}^{a}>S_{i j}^{a, \max } \\
0, & \text { caso contrário }
\end{array}\right.
$$

$S_{i j}^{a}$ : Fluxo potência aparente sobre a linha $i j$ da área $a$;

$S_{i j}^{a, \max }$ : Capacidade máxima de fluxo potência aparente sobre a linha $i j$ da área $a$.

B5. Restrição de acoplamento:

$$
h_{5}=\operatorname{Min} \sum\left(w_{e s p}^{a}-\sum_{j \in k a} w_{j}\right), \quad \forall a \in A
$$

\section{Restrições}

As restrições do problema de otimização multiobjetivo são as restrições de fluxo de carga:

$$
\begin{gathered}
P_{g i}^{a}-P_{L i}^{a}-P(\theta, V, t)_{i}^{a}=0, \quad \forall i \in N, \quad \forall a \in A \\
Q_{g i}^{a}-Q_{L i}^{a}-Q(\theta, V, t)_{i}^{a}+S_{H_{i}}^{a}=0, \quad \forall i \in N, \quad \forall a \in A
\end{gathered}
$$

Em que:

$P_{L i}^{a} \mathbf{e} Q_{L i}^{a}$ : Demanda de potência ativa e reativa na $i$-ésima barra da área $a$;

$P(V, \theta, t)_{i}^{a}$ : Injeção líquida de potências ativa na barra $i$, pertencente à área $a$; 
$\theta_{i}:$ Ângulo de fase na barra $i$

$t_{i}: i$-ésimo transformador com controle automático de taps;

$Q(V, \theta, t)_{i}^{a}:$ Injeção líquida de potências reativa na barra $i$, pertencente à área $a$;

$S_{H_{i}}^{a}$ : Banco de capacitor/reator shunts na $i$-ésima barra, pertencente à área $a$.

\subsection{AEMO Dedicado a Solução dos Sub- problemas Regionais de FPO}

Visando-se gerar um conjunto suficientemente diversificado de soluções não-dominadas e obter um bom desempenho, na solução de problemas complexos, o algoritmo proposto combina algumas estratégias, tais como:

- Codificação das variáveis de controle em base real;

- Elitismo;

- Desacoplamento implícito das variáveis do problema;

- Seleção e recombinação simultaneamente;

- Redução do conjunto de Pareto por clustering;

- Preservação da diversidade.

\subsubsection{Codificação e População Inicial}

Devido às características físicas do problema, para se obter um desempenho satisfatório do AEMO sob os aspectos da eficiência computacional e qualidade das soluções otimizadas fornecidas pelo algoritmo, é necessário gerar uma população inicial de boa qualidade. Os indivíduos (cromossomos) que compõem a população podem ser formados por $n$ subconjuntos de variáveis, representadas pelas variáveis de controle do problema em questão. A Figura 2 ilustra um indivíduo formado por quatro subconjuntos representados pelas as variáveis de controle contínuas $\left(\mathbf{u}_{C_{i}}\right)$ e discretas $\left(\mathbf{u}_{D_{i}}\right)$.

A potência ativa gerada $\left(P_{g i}\right)$ e as magnitudes de tensões $\left(V_{i}\right)$ são tratadas como variáveis de controle contínuas e geradas de forma pseudo-aleatória para atender a região de factibilidade destas variáveis. Além disso, a potência ativa gerada na área em análise deve suprir a sua demanda e suas perdas.

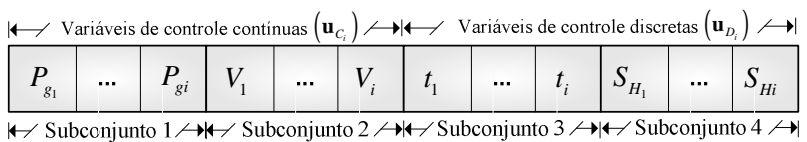

Figura 2: Estrutura do cromossomo da população.
Os transformadores com controle automático de taps $\left(t_{i}\right)$ e os bancos de capacitores e reatores shunts $\left(S_{H i}\right)$ são representados por valores discretos como segue:

Seja $n_{i}$ número aleatório inteiro no intervalo $\left[0, \ldots, M_{i}\right]$,

$$
\begin{aligned}
& M_{i}=\operatorname{int}\left(\frac{u_{D_{i}}^{a, \max }-u_{D_{i}}^{a, \min }}{\Delta \mathbf{u}_{D}}\right), \quad \forall i \in N, \quad \forall a \in A \\
& u_{D_{i}}^{a}=u_{D_{i}}^{a, \min }+n_{i} \cdot\left(\Delta \mathbf{u}_{D}\right), \quad \forall i \in N, \quad \forall a \in A
\end{aligned}
$$

Em que:

$\Delta \mathbf{u}_{D}:$ Tamanho do passo de discretização;

$u_{D_{i}}^{a, \text { min }}:$ Limite inferior das variáveis discretas na área $a$;

$u_{D_{i}}^{a, \max }$ : Limite superior das variáveis discretas na área $a$.

Tanto as variáveis contínuas, quanto as variáveis discretas são codificadas em base real satisfazendo a suas respectivas regiões de factibilidade. As vantagens deste sistema de codificação são armazenar uma maior quantidade de informações que a codificação binária para um cromossomo com a mesma dimensão e trabalhar com a representação real das variáveis do problema.

A partir das variáveis de controle contínuas e discretas estabelecidas para cada indivíduo calculam-se as restrições de fluxo de carga (23) e (24). Estas restrições são resolvidas através Método de Newton (Monticelli (1983)) e posteriormente calculam-se as funções objetivo (16)-(22).

\subsubsection{Estratégia Elitista}

A estratégia elitista, dentro do contexto multiobjetivo, deve ser expandida para o conjunto das soluções não-dominadas da população corrente. Este procedimento é fundamental na resolução de problemas multiobjetivos, uma vez que a solução destes problemas é na verdade um conjunto de soluções fronteira de Pareto-ótima. Após a classificação da população os pontos pertencentes à primeira fronteira $\left(F_{1}\right)$ são retirados da população e armazenados em um subconjunto elitista $(E)$, para que sejam utilizados no processo de recombinação com o objetivo de aumentar a pressão de seleção e ao mesmo tempo acelerar a convergência do algoritmo.

\subsubsection{Desacoplamento Implícito das Variáveis de Controle}

A estratégia de desacoplamento implícito tem por objetivo contemplar a solução de problemas que apresentam variáveis 
de controle conflitantes entre si. Na metodologia proposta as violações das restrições de desigualdades são tratadas como funções objetivo do problema e, ao atualizar um determinado conjunto de variáveis de controle através de recombinação ou mutação, um objetivo é melhorado em detrimento de outros. Desta forma, ao se sortear os indivíduos (cromossomo) para executar a recombinação ou mutação, deve-se verificar qual dos objetivos está sendo contemplado e efetuar a recombinação ou mutação considerando apenas o(s) subconjunto(s) de variáveis de controle que interferem diretamente neste(s) objetivo(s).

\subsubsection{Operadores Genéticos}

\section{a) Seleção e recombinação}

O procedimento de seleção adotado é o de torneio, na qual algumas soluções são aleatoriamente escolhidas da população e, com base em algum critério, a solução vencedora é então selecionada. Normalmente, o critério utilizado pela maioria dos algoritmos evolutivos mono-objetivo é o valor da função de aptidão, já para os algoritmos evolutivos multiobjetivos alguma estratégia de nicho é empregada de forma a modificar as aptidões reais dos indivíduos conforme a densidade de vizinhos no seu entorno.

Neste trabalho, o procedimento de torneio empregado é efetuado diretamente sobre as ordens (fronteiras) recebidas pelos indivíduos, deste modo, os indivíduos são selecionados não só pelas suas aptidões, mas sim pelas suas aptidões dentro do contexto multiobjetivo de dominância. Além disso, este procedimento é realizado em conjunto com o operador de recombinação de um único ponto.

Seja $\left\{N_{\text {pop }}\right\}$ o número máximo de indivíduos da população e $\{M\}$ um conjunto que contém as soluções $\left\{M / M \in N_{\text {pop }}\right.$ e $\left.M \notin E\right\}$ a serem utilizadas nos processos de seleção e recombinação, como segue:

i Selecionar por torneio um indivíduo pai, P1, do subconjunto $M$;

ii Selecionar aleatoriamente o segundo pai, P2, do subconjunto $E$;

iii Selecionar aleatoriamente um dos objetivos do problema e iniciar o processo de recombinação;

iv Gerar um número aleatório $r \in[0,1]$. Se $r<\operatorname{Pr}(\operatorname{Pr}$ é a probabilidade de recombinação), então obter aleatoriamente o ponto de recombinação. Caso contrário, voltar ao passo $i$;

v Se o objetivo escolhido no passo iii se referir ao custo da geração, então a recombinação de um único ponto será realizada considerando todos os subconjuntos de variáveis de controle (Figura 2). Caso contrário, o desacoplamento implícito das variáveis do problema será realizado;

vi Repetir os passos de $i$ à $v$ até que a nova população possua o número de indivíduos predefinido.

\section{b) Mutação}

A mutação é um operador de grande importância para a solução de problema do mundo real, pois introduz, aleatoriamente, novas informações na população, prevenindo a convergência prematura do algoritmo. A sequência deste procedimento é descrita abaixo:

i Gerar um número aleatório $m \in[0,1]$;

ii Se $m<\operatorname{Pm}$ (Pm é a probabilidade de mutação), então selecionar aleatoriamente um dos objetivos do problema, para realizar a mutação. Caso contrário, voltar ao passo $i$

iii Se o objetivo escolhido no passo ii for diferente do custo da geração, então deve-se realizar o desacoplamento implícito das variáveis do problema. Caso contrário, a mutação será efetuada em todos os subconjuntos das variáveis de controle (Figura 2);

iv Selecionar o ponto de mutação para a variável que sofrerá mutação;

v Trocar o valor atual da variável selecionada por um valor gerado, aleatoriamente, no domínio desta variável;

vi Repetir os passos $i$ à $v$ até que a nova população tenha o número de indivíduos predefinido.

\subsubsection{Redução do Conjunto de Pareto}

Em alguns problemas o conjunto Pareto-Ótimo pode ser extremamente grande. Nestes casos, a redução do conjunto de soluções não-dominadas sem a degradação das características da fronteira Pareto-ótima é indispensável. Neste trabalho, para a redução do número de soluções contidas no conjunto Pareto-ótimo utilizou-se a técnica denominada clustering (Morse (1980)). Esta técnica particiona o conjunto de soluções dominantes em $N$ grupos (clusters) de acordo com a proximidade das soluções. Para cada cluster, selecionase uma solução representativa (centróide) e as soluções restantes são descartadas. A distância entre quaisquer dois elementos do conjunto de soluções não-dominadas é encontrada através da distância euclidiana (Zitzler e Thiele (1999)). 


\subsubsection{Preservação da Diversidade}

Geralmente para preservar a diversidade da população em algoritmos evolutivos multiobjetivos utiliza-se a técnica de $n i$ ching (Goldberg (1989); Deb et al. (2000)). A técnica de niching consiste na divisão da população em espécies (que reúnem indivíduos com características semelhantes) para reduzir a competição por recursos e criar subpopulações estáveis, cada uma delas concentrada em um nicho do espaço de busca.

Neste trabalho, um esquema de niching utilizando o mecanismo de sharing foi adicionado ao método proposto. O mecanismo de sharing trabalha alterando a função de avaliação de cada elemento da população de acordo com o número de indivíduos semelhantes a ele na população (Mathfound (2000)). Porém, ao contrário do esperado, seus resultados não melhoraram significativamente a diversidade das soluções não dominadas encontradas. Dessa forma, para preservar a diversidade na população, além do mecanismo de sharing utilizaram-se as taxas de recombinação $(P r)$ e mutação $(P m)$ atualizadas de forma adaptativa, como segue:

$$
\begin{aligned}
& P r=\left[\operatorname{Pr}^{\text {max }}-i g \cdot\left(\operatorname{Pr}^{\text {max }}-\operatorname{Pr}^{\text {min }}\right)\right] / n \max \\
& P m=\left[P m^{\text {min }}+i g \cdot\left(P m^{\text {max }}-P m^{\text {min }}\right)\right] / n \max
\end{aligned}
$$

Em que:

$P r^{\min }, \operatorname{Pr}^{\max }$ : Limites inferiores e superiores referentes à taxa de recombinação;

$P m^{\text {min }}, P m^{\text {max }}$ : Limites inferiores e superiores referentes à taxa de mutação;

$i g:$ Índices das gerações;

nmax: Número máximo de gerações.

\subsection{Técnica Fuzzy para Determinar a Me- Ihor Solução Compromisso}

Os algoritmos multiobjetivos fornecem um conjunto de grande dimensão de soluções aceitáveis. Tais soluções estão distribuídas em diversas fronteiras, e conforme já mencionado, todos os pontos em uma fronteira particular possuem o mesmo grau de dominância, sendo os pontos da primeira fronteira os mais aptos, porque estão associados aos pontos dominantes da população.

Na solução do problema de FPO é desejável encontrar uma solução para o qual os valores de todas as funções objetivo são considerados aceitáveis pelo projetista ou decisor. Neste trabalho, para determinar uma solução particular, após a convergência do algoritmo as soluções não-dominadas pertencentes à primeira fronteira de Pareto-ótima (soluções analisadas em relação às funções objetivo (16), (17) e as funções objetivo referentes às violações das restrições de desigualdades (18)-(22)) são analisadas em relação à redução das perdas de potência ativa nas linhas de transmissão e despacho ótimo de potências ativa e reativa. Para esta finalidade aplicam-se os conceitos de dominância (Seção 3) para as funções objetivo (16), (17) e (19) para obter a primeira fronteira de Pareto-ótima. Após obter as soluções não-dominadas pertencentes à primeira fronteira de Pareto-ótima $\left(F_{1}\right)$ aplica-se um mecanismo de decisão, baseado na teoria dos conjuntos fuzzy (Abido (2006); Srinivasan et al. (1994); Dhillon et al. (1993); Dhillon et al. (2002)).

Os conjuntos fuzzy são definidos através de equações denominadas funções de pertinência $\left(\mu_{i}\right)$. Estas funções representam o grau de pertinência no conjunto fuzzy usando valores entre 0 e 1 (Dhillon et al. (1993)). Os valores das funções de pertinência indicam o grau de satisfação das funções objetivos do problema. A equação abaixo expressa a função de pertinência que é definida como em (Dhillon et al. (1993)).

$$
\begin{gathered}
\mu_{i}=\left\{\begin{array}{lc}
1 & f_{i}(x) \leq f_{i}^{\min }(x) \\
0 & f_{i}(x) \geq f_{i}^{\max }(x) \\
\frac{f_{i}^{\max }(x)-f_{i}(x)}{f_{i}^{\max }(x)-f_{i}^{\min }(x)} & f_{i}^{\min }(x) \leq f_{i}(x) \leq f_{i}^{\max }(x)
\end{array}\right. \\
i=1, \ldots, N_{o b j}
\end{gathered}
$$

sendo:

$f_{i}^{\min }(x) \mathbf{e} f_{i}^{\max }(x)$ : Valores mínimos e máximos da $i$-ésima função objetivo.

Seja $N_{d o m}$ o número de soluções não-dominadas da primeira fronteira de Pareto, então, para cada solução não-dominada $k\left(k \in N_{d o m}\right)$ a função de pertinência é normalizada como:

$$
\mu^{k}=\frac{\sum_{i=1}^{N_{o b j}} \mu_{i}^{k}}{\sum_{k=1}^{N_{d o m}} \sum_{i=1}^{N_{o b j}} \mu_{i}^{k}}
$$

A melhor solução compromisso é dada por $\gamma=$ $\max \left\{\mu^{k}\right\}, \quad k=1,2, \ldots, N_{\text {dom }}$. 
Para estabelecer os valores de $f_{i}^{\min }(x)$ e $f_{i}^{\max }(x)$ inicialmente as soluções não-dominadas na primeira fronteira de Pareto são ordenadas. A pior solução encontrada, para a $i$-ésima função objetivo, é denominada $f_{i}^{\max }(x)$. Quanto aos valores mínimos considera-se $f_{i}^{\min }(x)=0$, se a $i$-ésima função objetivo referir-se aos custos das perdas nas linhas de transmissão ou às infactilidades das restrições de desigualdade. Se a $i$-ésima função objetivo referir-se ao custo da geração, então $f_{i}^{\min }(x)$ será o menor valor encontrado no conjunto de soluções da primeira fronteira de Pareto para esta função objetivo.

\subsection{Diagrama de Bloco do Algoritmo Pro- posto}

Na figura 3 apresenta-se o diagrama de blocos do algoritmo proposto para a solução do problema de FPO descentralizado.

\section{RESULTADOS}

O desempenho e a robustez da metodologia proposta foram avaliados utilizando os sistemas teste RTS-96 (Grigg et al. (1999)) e IEEE-354 (Aguado e Quintana (2001)). Os dois sistemas teste utilizados para as análises da metodologia são constituídos de três áreas interconectadas (Área 1, Área 2 e Área 3) e as condições de simulações para ambos os sistemas são:

- Limites das magnitudes de tensões: $V_{i}^{a, \min }=0,94 \mathrm{pu}$ e $V_{i}^{a, \min }=1,06 p u$

- Tamanhos do passo para as variáveis discretas: $\Delta \mathbf{u}_{D}=$ 0, 01 pu para os transformadores com controle automático de taps e $\Delta \mathbf{u}_{D}=0,125 \mathrm{pu}$ para os capacitores/reatores shunt;

- Limites das taxas de recombinação e mutação: $P r^{\min }=0,001 ; \operatorname{Pr}^{\max }=0,9 ; P^{\min }=$ 0,$01 ; P m^{\max }=0,5$;

- Critério de parada para o AEMO: número máximo de gerações $($ max $)$.

Nas tabelas 1 e 2 apresentam-se os custos da geração de potência ativa e das perdas nas linhas de transmissão e as violações na geração de potência reativa para os arquivos de dados originais para os sistemas RTS-96 e IEEE-354, referenciados neste trabalho como caso base. Estes resultados são obtidos através de um programa de fluxo de carga convencional utilizando o método de Newton.

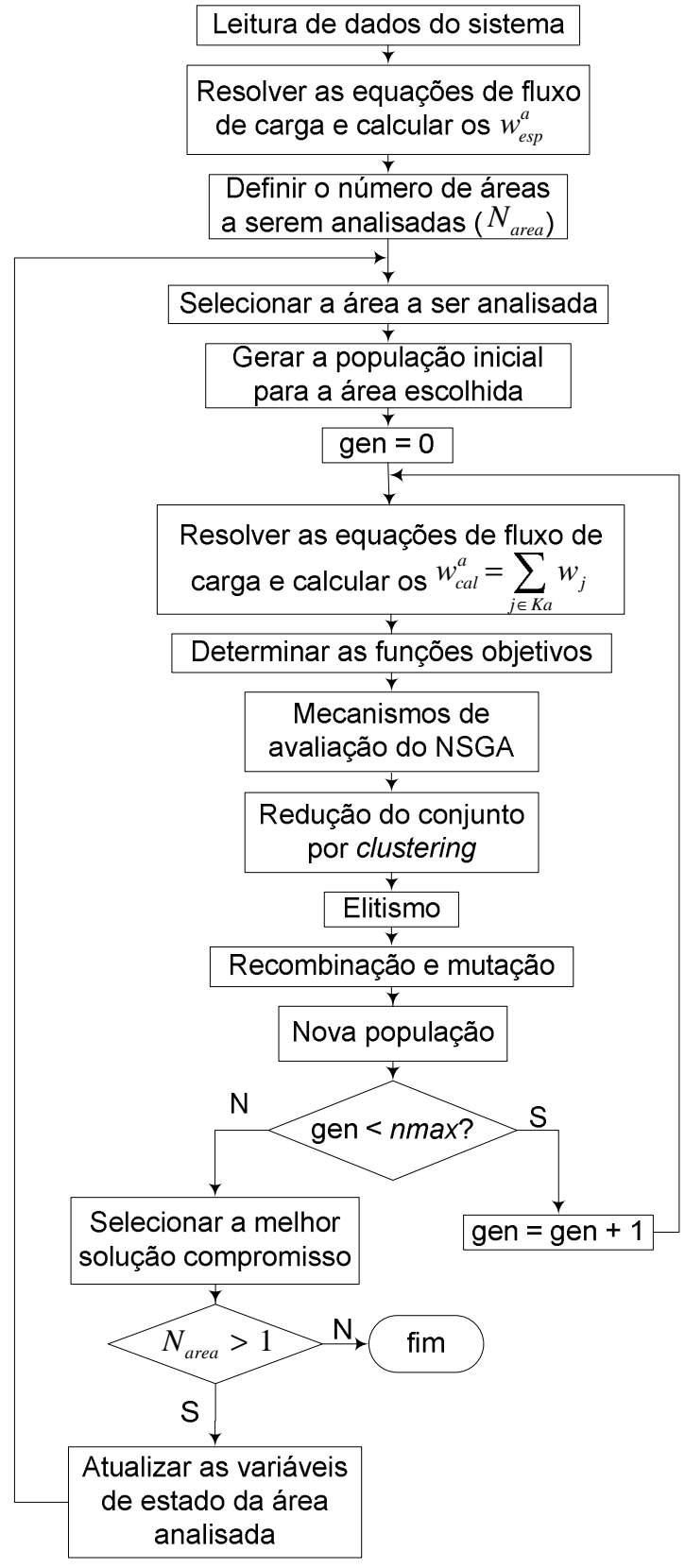

Figura 3: Diagrama de blocos da técnica de solução aplicada ao problema de FPO descentralizado.

Tabela 1: Resultados do caso base - RTS-96.

\begin{tabular}{|c|c|c|r|}
\hline $\begin{array}{c}\text { Caso base } \\
\text { (fluxo de carga) }\end{array}$ & $\begin{array}{c}f_{1} \\
(\$ / \mathrm{hr})\end{array}$ & $\begin{array}{c}f_{2} \\
(\$ / \mathrm{hr})\end{array}$ & $\begin{array}{c}h_{2} \\
(\mathrm{MVAr})\end{array}$ \\
\hline Área 1 & $42.825,39$ & $1.019,37$ & 41,02 \\
\hline Área 2 & $42.816,14$ & $1.014,64$ & 38,07 \\
\hline Área 3 & $42.568,90$ & $1.019,75$ & 37,69 \\
\hline
\end{tabular}


Tabela 2: Resultados do caso base - IEEE-354.

\begin{tabular}{|c|c|c|r|}
\hline $\begin{array}{c}\text { Caso base } \\
\text { (fluxo de carga) }\end{array}$ & $\begin{array}{c}f_{1} \\
(\$ / \mathrm{hr})\end{array}$ & $\begin{array}{c}f_{2} \\
(\$ / \mathrm{hr})\end{array}$ & $\begin{array}{c}h_{2} \\
(\mathrm{MVAr})\end{array}$ \\
\hline Área 1 & $131.375,20$ & $3.478,82$ & 375,75 \\
\hline Área 2 & $131.322,00$ & $3.470,89$ & 336,56 \\
\hline Área 3 & $131.322,00$ & $3.704,11$ & 397,48 \\
\hline
\end{tabular}

\subsection{Sistema RTS-96}

Este sistema é constituído de 72 barras e 119 linhas de transmissão, sendo que 05 linhas destas são as linhas que fazem o acoplamento entre as 03 áreas que constituem o sistema. Cada área deste sistema contém 05 transformadores com controle automático de taps e 01 reator shunt. Em todas as simulações, o tamanho da população foi adotado como 400 indivíduos e o número máximo de gerações igual a 500 gerações.

As figuras 4, 5 e 6 ilustram a primeira fronteira de Paretoótima e a melhor solução compromisso obtida nas análises dos subproblemas regionais de FPO para as áreas 1, 2 e 3 respectivamente. Nestas figuras pode-se observar o espaço das soluções não-dominadas na primeira fronteira de Paretoótima, considerando se a redução nos custos das perdas nas linhas de transmissão, o custo total da geração de potência ativa e a minimização das infactibilidades ocorridas na geração de potência reativa.

Analisando-se as Figuras 4, 5 e 6 conclui-se que o algoritmo convergiu para uma fronteira de Pareto-ótima bem distribuída na qual as soluções não-dominadas estão ao longo de uma curva claramente identificável. Os custos da geração e das perdas, assim como as violações na geração de potência reativa para a melhor solução compromisso são apresentados na Tabela 3.

Tabela 3: Melhor solução compromisso obtida para cada subproblema regional de FPO.

\begin{tabular}{|c|c|r|r|}
\hline $\begin{array}{c}\text { Subproblemas } \\
\text { regionais de } \\
\text { FPO }\end{array}$ & $\begin{array}{c}f_{1} \\
(\$ / \mathrm{hr})\end{array}$ & $\begin{array}{c}f_{2} \\
(\$ / \mathrm{hr})\end{array}$ & $\begin{array}{c}h_{2} \\
(\mathrm{MVAr})\end{array}$ \\
\hline Área 1 & $42.591,37$ & 876,58 & 0,00 \\
\hline Área 2 & $42.578,83$ & 879,28 & 0,00 \\
\hline Área 3 & $42.276,40$ & 883,84 & 0,00 \\
\hline
\end{tabular}

Comparando as Tabelas 1 e 3 observa-se que as violações na geração de potência reativa, para a área sob análise, foram reduzidas em $100 \%$. Considerando-se um nível de carregamento nominal verifica-se que em cada hora obtém-se uma redução nas perdas das linhas de transmissão do sistema elétrico de aproximadamente 14\%. Esta redução representa um ganho médio de aproximadamente \$99.374,00 ao mês. Quanto aos custos da geração de potência ativa a redução foi

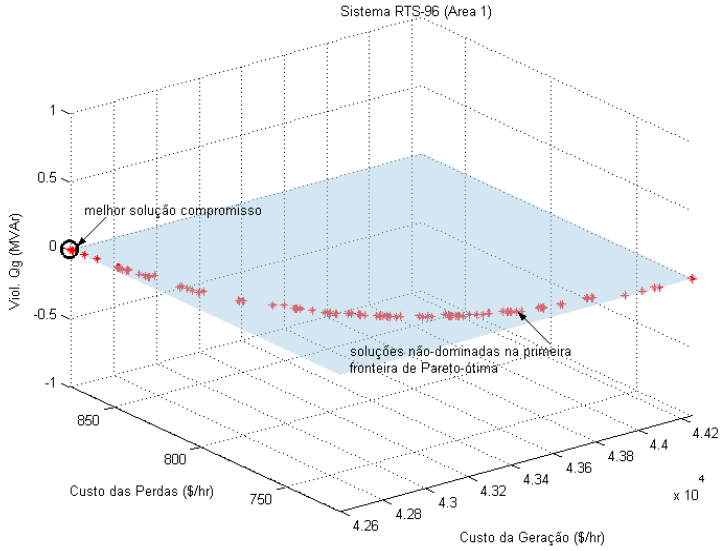

Figura 4: Soluções não-dominadas na $1^{a}$ fronteira de Paretoótima para a área 1.

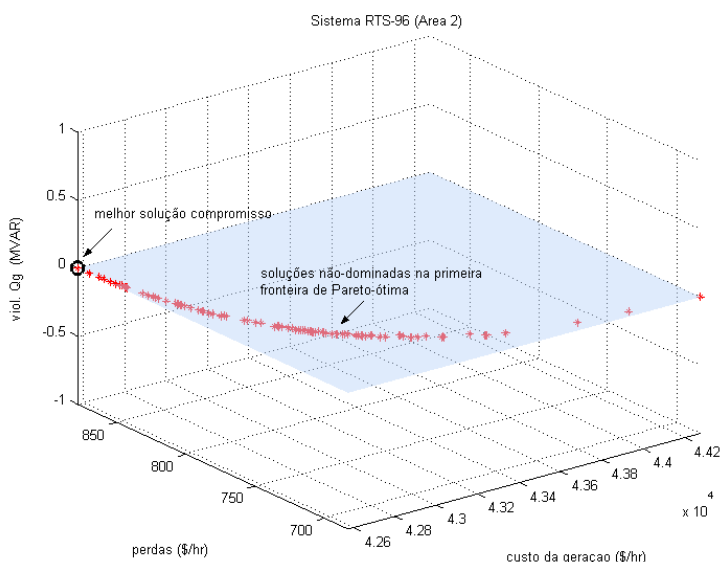

Figura 5: Soluções não-dominadas na $1^{a}$ fronteira de Paretoótima para a área 2.

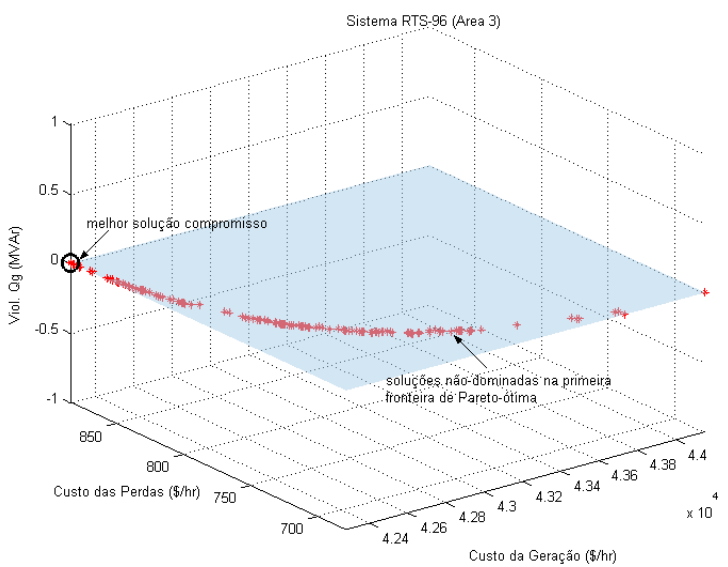

Figura 6: Soluções não-dominadas na $1^{a}$ fronteira de Paretoótima para a área 3. 
de aproximadamente $0,5 \%$. Este fato se justifica pelo conflito entre os objetivos e também pelo conflito entre as restrições operacionais e a função objetivo relacionada com o despacho econômico de potência ativa. Mas ao analisar o ganho médio obtido na redução dos custos da geração de potência ativa, no decorrer de um mês, o ganho médio em cada subproblema regional de FPO foi de \$183.320,00. Somando-se os ganhos nos custos da geração e das perdas nas linhas de transmissão totaliza-se um ganho total de aproximadamente \$ 282.690,00 ao mês, em cada área analisada. Além dos ganhos financeiros a aplicação da metodologia propiciou uma operação mais segura, capaz de atender todas as restrições impostas para o problema.

Na Tabela 4 encontram-se relacionados os resultados da melhor solução compromisso para as análises sucessivas dos subproblemas regionais de FPO.

Os resultados apresentados na Tabela 4 foram obtidos analisando-se inicialmente o subproblema regional de FPO referente à Área 1. Após a convergência do algoritmo os valores obtidos para as variáveis de controle desta área são atualizados na base de dados do sistema e um novo subproblema é selecionado para ser otimizado, neste caso, escolheu-se o subproblema regional de FPO referente à Área 2. Este processo é repetido até que todas as áreas do sistema de potência interconectado sejam analisadas.

Comparando-se os resultados apresentados na Tabela $1 \mathrm{com}$ os resultados obtidos após resolver o subproblema regional de FPO da Área 1 (Tabela 4) observa-se que ao otimizar a Área 1 os resultados das Áreas 2 e 3 permaneceram inalterados. Além disso, observando-se os resultados numéricos de cada subproblema regional de FPO analisado (Tabela 4) têm-se que ao otimizar uma determina área, as áreas que não estão sendo analisadas têm as suas condições operacionais inalteradas. As pequenas variações nos custos das perdas e

Tabela 4: Melhor solução compromisso obtida para as análises sucessivas dos subproblemas regionais de FPO.

\begin{tabular}{|c|c|c|c|c|c|}
\hline $\begin{array}{l}\text { Subproblemas } \\
\text { regionais de } \\
\text { FPO analisados }\end{array}$ & Áreas & $\begin{array}{c}f_{1} \\
(\$ / \mathrm{hr})\end{array}$ & $\begin{array}{c}f_{2} \\
(\$ / \mathrm{hr})\end{array}$ & $\begin{array}{c}h_{2} \\
\text { (MVAr) }\end{array}$ & \\
\hline \multirow{3}{*}{ Área 1} & 1 & $42.552,20$ & 921,01 & 0,00 & $\rightarrow$ Área analisada \\
\hline & 2 & $42.816,14$ & $1.015,69$ & 38,59 & \multirow{2}{*}{$\begin{array}{c}\text { Áreas não analisadas } \\
\text { (valores iguais ao } \\
\text { caso base (Tabela 2) }\end{array}$} \\
\hline & 3 & $42.568,90$ & $1.025,32$ & 37,69 & \\
\hline \multirow{3}{*}{$\begin{array}{c}\text { Área } 2 \\
\text { (analisada após } \\
\text { a otimização da } \\
\text { área 1) }\end{array}$} & 1 & $42.552,20$ & 905,03 & 0,00 & \multirow{2}{*}{ >Áreas analisadas } \\
\hline & 2 & $42.635,13$ & 915,82 & 0,00 & \\
\hline & 3 & $42.568,90$ & 1025,32 & 47,78 & $\rightarrow$ Área não analisada \\
\hline \multirow{3}{*}{$\begin{array}{c}\text { Área } 3 \\
\text { (analisada após } \\
\text { a otimização } \\
\text { das áreas } 1 \text { e } 2 \text { ) }\end{array}$} & 1 & $42.552,20$ & 902,64 & 0,00 & \multirow{3}{*}{ Áreas analisadas } \\
\hline & 2 & $42.635,13$ & 914,64 & 0,00 & \\
\hline & 3 & $42.279,44$ & 886,07 & 0,00 & \\
\hline
\end{tabular}

nos valores das violações de potência reativa decorrem da não-linearidade do problema. Desta forma, pode-se concluir que a restrição de acoplamento imposta ao problema foi devidamente atendida.

\subsection{Sistema IEEE-354}

Este sistema é baseado no sistema teste IEEE-118 (Lebow, (1984)) e possui 354 barras e 543 linhas de transmissão, sendo que 06 linhas são as linhas que fazem o acoplamento entre as 03 áreas que constituem o sistema. Cada área deste sistema contém 09 transformadores com controle automático taps e 14 capacitores e reatores shunt. Em todas as simulações, o tamanho da população foi adotado como 900 indivíduos e o número máximo de gerações igual a 1800 gerações.

As figuras 7, 8 e 9 ilustram a primeira fronteira de Paretoótima e a melhor solução compromisso obtida nas análises dos subproblemas regionais de FPO para as áreas 1, 2 e 3 respectivamente. Nestas figuras pode-se observar o espaço das soluções não-dominadas na primeira fronteira de Pareto-ótima para cada subproblema regional de FPO, considerando-se a minimização dos custos das perdas de potência ativa nas linhas de transmissão, a minimização do custo total da geração de potência ativa e a minimização das violações de potência reativa gerada a área em análise $\left(\Delta Q_{g i}^{a}\right)$.

A média da redução dos custos das perdas de potência ativa nos subproblemas regionais de FPO foi de aproximadamente $59 \%$, quando comparada com o valor das perdas para os valores das variáveis de controle do arquivo original (Tabela 2). Para obter uma condição de operação mais segura, ou seja, manter as magnitudes de tensões e fluxos de potência nas linhas de transmissão dentro dos limites preestabelecidos, não foi possível reduzir os custos na geração de potência ativa em nenhum dos subproblemas regionais de FPO analisado.

Analisando a Tabela 2 conclui-se que no estado de operação com as variáveis de controle ajustadas de acordo com o arquivo de dados original (Lebow (1984)), este sistema necessita de suporte de potência reativa que supere sua capacidade de geração de reativos. Este ponto de operação inicial causa dificuldades para se encontrar soluções sem violações na geração de potência reativa e, consequentemente, algumas soluções contidas na fronteira de Pareto-ótima, mostrada nas Figuras 7, 8 e 9, apresentam violações nesta restrição. Ao aplicar as funções de pertinências para determinar a solução não-dominada que melhor represente o subproblema regional de FPO as soluções mais violadas são excluídas e uma solução que apresente pequenas violações é selecionada.

Analisando-se as Figuras 7, 8 e 9 verifica-se que em todas as simulações realizadas o algoritmo convergiu para uma 


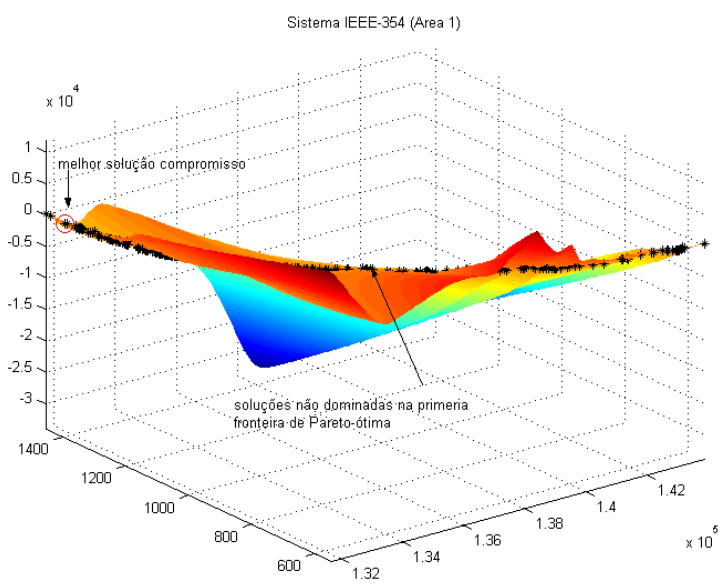

Figura 7: Soluções não-dominadas na $1^{a}$ fronteira de Paretoótima para a área 1.

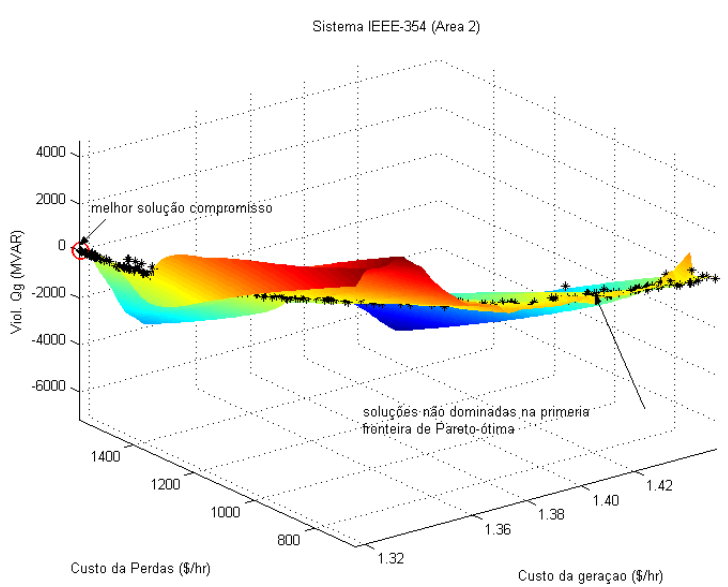

Figura 8: Soluções não-dominadas na $1^{a}$ fronteira de Paretoótima para a área 2.

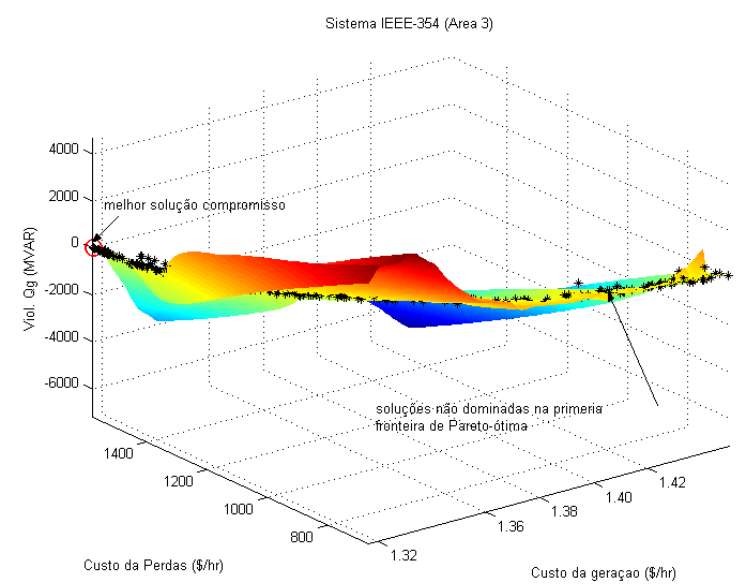

Figura 9: Soluções não-dominadas na $1^{a}$ fronteira de Paretoótima para a área 3.
Tabela 5: Melhor solução compromisso obtida para cada subproblema regional de FPO

\begin{tabular}{|c|c|c|r|}
\hline $\begin{array}{c}\text { Subproblemas } \\
\text { regionais de } \\
\text { FPO }\end{array}$ & $\begin{array}{c}f_{1} \\
(\$ / h r)\end{array}$ & $\begin{array}{c}f_{2} \\
(\$ / h r)\end{array}$ & $\begin{array}{c}h_{2} \\
(\mathrm{MVAr})\end{array}$ \\
\hline Área 1 & $131.735,86$ & $1.393,56$ & 23,13 \\
\hline Área 2 & $131.858,77$ & $1.351,28$ & 0,00 \\
\hline Área 3 & $131.669,15$ & $1.583,92$ & 0,68 \\
\hline
\end{tabular}

fronteira de Pareto-ótima bem distribuída na qual as soluções não-dominadas estão ao longo de uma curva claramente identificável.

Na Tabela 5 apresentam-se as melhores soluções compromisso obtidas nas análises dos subproblemas regionais de FPO e aplicação da teoria dos conjuntos fuzzy (seção 4.3).

Nas análises com os subproblemas regionais de FPO, referentes às áreas 2 e 3 os custos da geração de potência ativa foram relativamente maiores quando comparados com o caso base (Tabela 2). Este fato decorre das características do sistema e não afeta o desempenho da metodologia, pois ao somar os custos da geração com os custos das perdas observase que a metodologia apresenta uma operação mais segura e ainda obtém-se a redução dos custos operacionais de cada uma das empresas de geração. Esta análise está esquematizada na Figura 10.

Os limites mínimos e máximos obtidos nas análises de cada subproblema de FPO e para o caso base são apresentados na Tabela 6. Verifica-se nesta tabela que o perfil das magnitudes das tensões é mantido dentro dos limites preestabelecidos $\left(V_{i}^{a, \min }=0,94 p u\right.$ e $\left.V_{i}^{a, \min }=1,06 p u\right)$.

\begin{tabular}{|c|c|c|c|c|c|}
\hline $\begin{array}{l}\text { Caso base } \\
\text { (método de } \\
\text { Newton) }\end{array}$ & \multicolumn{2}{|c|}{$\begin{array}{c}f_{1}+f_{2} \\
(\$ / \mathrm{hr})\end{array}$} & \multicolumn{2}{|c|}{$\begin{array}{l}\text { Subproblemas } \\
\text { regionais de } \\
\text { FPO }\end{array}$} & $\begin{array}{c}f_{1}+f_{2} \\
(\$ / \mathrm{hr})\end{array}$ \\
\hline Área 1 & \multicolumn{2}{|c|}{$134.852,02$} & & ea 1 & $133.129,42$ \\
\hline Área 2 & \multicolumn{2}{|c|}{$134.792,82$} & & 2 & $133.210,05$ \\
\hline Área 3 & \multicolumn{2}{|c|}{$135.026,11$} & & ea 3 & $133.253,07$ \\
\hline \multicolumn{2}{|c|}{$\begin{array}{c}\text { Subproblemas } \\
\text { regionais de } \\
\text { FPO }\end{array}$} & \multicolumn{2}{|c|}{$\begin{array}{l}\text { Lucros por hora } \\
\qquad(\$ / h r)\end{array}$} & \multicolumn{2}{|c|}{$\begin{array}{l}\text { Lucro ao mês } \\
\qquad \text { (\$/mês) }\end{array}$} \\
\hline \multicolumn{2}{|c|}{ Área 1} & \multicolumn{2}{|c|}{$1.722,60$} & \multicolumn{2}{|c|}{$1.240 .272,00$} \\
\hline \multicolumn{2}{|c|}{ Área 2} & \multicolumn{2}{|c|}{$1.582,77$} & \multicolumn{2}{|c|}{$1.139 .594,40$} \\
\hline \multicolumn{2}{|c|}{ Área 3} & \multicolumn{2}{|c|}{$1.773,04$} & \multicolumn{2}{|c|}{$1.276 .588,80$} \\
\hline
\end{tabular}

Figura 10: Lucros obtidos em cada subproblema regional de FPO. 
Tabela 6: Limites mínimos e máximos obtidos para cada subproblema regional de FPO e para o caso base

\begin{tabular}{|c|c|c||c|c|}
\hline $\begin{array}{c}\text { Subproblemas } \\
\text { regionais de } \\
\text { FPO }\end{array}$ & $V^{\text {min }}$ & $V^{\text {max }}$ & \multicolumn{2}{|c|}{$\begin{array}{c}\text { Caso base } \\
\text { (fluxo de carga) }\end{array}$} \\
\hline Área 1 & 0,960 & 1,032 & $V^{\text {min }}$ & $V^{\text {max }}$ \\
\hline Área 2 & 0,953 & 1,040 & 0,930 & 1,050 \\
\hline Área 3 & 0,950 & 1,023 & 0,930 \\
\hline
\end{tabular}

\subsection{Discussão dos resultados}

O AEMO implementado convergiu para soluções de boa qualidade, ou seja, alteraram-se os controles de potência reativa disponíveis, melhorando o perfil de tensão. Ressalta-se que as magnitudes de tensões $\left(h_{3}\right)$ permaneceram dentro dos limites estabelecidos para todas as barras (referência, geração e carga) dos sistemas testados. A restrição de fluxo nas linhas $\left(h_{4}\right)$ e a capacidade de geração de potência ativa $\left(h_{1}\right)$ foram devidamente atendidas nas simulações com os dois sistemas teste.

Os resultados numéricos apresentados nas seções 5.1 e 5.2 evidenciam o potencial da metodologia e da técnica de solução, para a resolução de problemas de FPO descentralizado. Os testes mostram que a metodologia pode ser aplicada no setor elétrico reestruturado, na qual os serviços de geração e transmissão são oferecidos por diferentes empresas, localizadas em diferentes regiões geográficas e elétricas.

Nas simulações realizadas foram utilizadas diferentes sementes para a geração da população inicial e operadores genéticos. Assim, para uma mesma área, diferentes sementes geram diferentes despachos de geração e perfis de tensão, mas independente da semente utilizada, o algoritmo converge para soluções semelhantes e de boa qualidade. Este fato pode ser comprovado ao analisar os resultados mostrados na Tabela 3 com os resultados apresentados na Tabela 4. Os resultados numéricos mostrados na Tabela 4 foram obtidos através de sucessivas análises e com sementes distintas das utilizadas nas simulações de cada subproblema mostrado na Tabela 3. Mas se as sementes fossem as mesmas os resultados obtidos para os dois casos seriam exatamente os mesmos.

As funções de pertinência (seção 4.3) são usadas para avaliar cada solução contida na primeira fronteira de Pareto-ótima e a solução com maior valor de função de pertinência é denominada melhor solução compromisso. Analisando a melhor solução compromisso mostrada nas figuras 4, 5 e 6 observase que os custos da geração de potência ativa foram priorizados em relação aos custos das perdas nas linhas de transmissão. Pois ao longo da fronteira de Pareto-ótima existem soluções que apresentam menor custo de perdas, do que o escolhido, mas estas soluções apresentam maiores custo de geração. Por outro lado, embora as soluções indicadas como melhor solução compromisso tenha custos de perdas maiores do que as outras soluções contidas na primeira fronteira de Pareto-ótima, estas soluções estão com as perdas nas linhas de transmissão significativamente reduzidas, quando comparadas com o caso base.

\section{CONCLUSÕES}

Neste trabalho um procedimento de decomposição foi aplicado ao problema de FPO multiárea para definir vários subproblemas regionais de FPO, isto é, um subproblema de FPO para cada uma das áreas de um sistema de potência interconectado. Através da decomposição do problema de FPO multiárea foi possível analisar as áreas do sistema de potência interconectado independentemente, entretanto nenhuma transação bilateral entre as áreas ou fluxo de potência específico entre as linhas de ligação sejam especificados.

As análises realizadas fornecem como resultados soluções ótimas ou de boa qualidade para todas as áreas do sistema de energia elétrica que podem ser consideradas uma empresa de energia elétrica independente ou uma área de grande interesse dentro de uma empresa de grande porte. Estas soluções permitem atender diferentes objetivos dependendo das necessidades do usuário tais como: minimização das perdas de potência ativa nas linhas de transmissão, despacho ótimo de potências ativa e reativa e ajustes dos controles de tensão enquanto atende um conjunto de restrições físicas, operacionais e de acoplamento. Consequentemente, a metodologia apresentada é muito útil para a nova estrutura competitiva do setor elétrico na qual deseja-se oferecer os serviços a custo mínimos, mantendo os padrões de qualidade e segurança e a independência entre as empresas de energia elétrica.

\section{AGRADECIMENTOS}

Os autores agradecem a Fundação de Ensino Pesquisa e Extensão de Ilha Solteira - FEPISA (Processo 001/2007), a Fundação de Apoio ao Desenvolvimento e Tecnologia do Estado de Mato Grosso do Sul - FUNDECT e CNPq (Processo 350226/2007-5) pelo financiamento deste projeto de pesquisa.

\section{REFERÊNCIAS}

Abido, M. A., (2006). "Multiobjective evolutionary algorithms for electric power dispatch problem;" IEEE Trans. On Evolutionary Computations, vol. 10, no. 3, pp. 315-329.

Aguado, J. A. and Quintana, V. H. (2001). "Inter-utilities power-exchange coordination: A market-oriented ap- 
proach;” IEEE Trans. Power Syst., vol. 16, pp.513-519.

Aguado, J. A.; Quintana, V. H and Conejo, A. J. (1999). "Optimal power flows of interconnected power systems;" in IEEE Power Eng. Soc. Summer Meeting, vol. 2, pp. 814-819.

Bakirtzis, A. G. and Biskas, P. N. (2003). "A decentralized solution to the DC-OPF of interconnected power systems;" IEEE Trans. Power Syst., vol. 18, pp.1007-1013.

Baldick, R.; Kim, B. H.; Chase C. and Luo, Y. (1999). "A fast distributed implementation of optimal power flow;" IEEE Trans. Power Syst., vol. 14, no. 3, pp.858-863.

Biskas, P. N.; Bakirtzis, A. G.; Macheras, N. I and Pasialis, N. K. (2005): "A decentralized implementation of DC optimal power on a network of computers;" IEEE Trans. Power Syst., vol. 20, pp.25-33.

Carpentier, J. L., (1962). “Contribution a l'etude du dispatching economique. Bull-Soc. Fr Elec., Ser. B3, 431447.

Deb, K.; Pratap, A.; Agarwal, S. and Meyarivan, T., (2000). "A fast and elitist non dominated sorting genetic algorithm for multiobjective optimization: NSGA-II;" Kan Gal report.

Dhillon, J. S.; Parti, S. C. and Kothari, D. P. (1993). "Stochastic economic emission load dispatch," Electric Power Syst. Res., vol. 26, pp. 186-197.

Dhillon, J. S.; Parti, S. C. and Kothari, D. P. (2002). "Fuzzy decision making in stochastic multiobjective short-term hydrothermal scheduling," IEE Proc.-C, 149, vol.2, pp. 191-200.

Dommel, H. W. and Tinney, W. F., (1968). "Optimal power flow solutions;" IEEE Trans. on Power Apparatus and Systems, no. 87, pp. 1866-1876.

Fonseca, C M. and Fleming, P J. (1998). "Multiobjective optimization and multiple constraint handling with evolutionary algorithms - Part I: A Unified formulation;" IEEE Trans. on Systems, Man, and Cybernetics - Part A: Systems and Humans, vol. 28, pp.26-37.

Goldberg, D. E. (1989). "Genetic algorithms in search optimization and machine learning;" Reading Massachusetts Addison-Wesley.

Grigg, C.; Wong, P.; Albrecht, P.; Allan, R.; Bhavaraju, M.; Billinton, R.; Chen, Q.; Fong, C.; Haddad, S.; Kuruganty, S.; Li, W.; Mukerji, R.; Patton, D.; Rau, N.; Reppen, D.; Schneider, A.; Shahidehpour, M.; Singh, C. (1999). "The reliability test system-1996;" IEEE Trans. Power Syst, vol. 14, pp.1010-1020.
Kim, B. H. and Baldick, R. (1997). "Coarse-grained distributed optimal solution flow;" IEEE Trans. Power Syst., vol. 12, no. 2, pp.932-939.

Lebow, W. M.; Mehra, R. K.; Nadira, R.; Rouhani, R. and Usoro, P.B. (1984). "Optimization of Reactive VoltAmperes (VAR) Sources in System Planning;"EPRI Report El-3729, vol I, Project 2109-1.

Mathfound, S. W. (2000). "Niching methods, In: Back, T.; Fogel, D.B.; Michalewicz (Eds.)," Evolutionary Computation 2, pp 87-92, Institute of Physics Publishing.

Monticelli, A., (1983). "Fluxo de carga em redes de energia elétrica;" Editora Edgard Blücher Ltda.

Morse, J. N. (1980). "Reducing the size of nondominated set: Pruning by clutering;" Comput. Oper. Res., vol. 7, no. $1-2$, pp.55-66.

Nogales, F. J.; Prieto, F. J. and Conejo, A. J. (1999). "Multi-area AC optimal power flow: A new decomposition approach;" in Proc. Power Syst. Comput. Conf.; Trondheim, Norway, pp.1201-1206.

Srinivas, N. and Deb, K. (1995). "Multi-objective function optimization using non-dominated sorting genetic algorithm;" Evolutionary Computation, vol. 2, no. 3, pp. 221-248.

Srinivasan, D.; Chang, C. S. and Liew, A. C. (1994). "Multiobjective generation schedule using fuzzy optimal search technique", Proc. Inst. Elect. Eng.-Gen. Transm. Dist., vol. 141, pp. 231-241.

Veldhuizen, D. A. V. and Lamont, G. B. (2000). "Multiobjective evolutionary algorithm: Analyzing the state-ofthe-art;" Evol. Comput., vol. 8, no. 2, pp. 125-147.

Yu, C. W. and David, A. K. (1996). "Transmission cost allocation in multi-area power pool operation;" IEE Power System Control and Management, Conference Publication, No. 421, pp. 16-18.

Zitzler, E. and Thiele, L. (1999). "Multiobjective evolutionary algorithm: a comparative case study and the Strength Pareto approach;" IEEE Transaction on Evolutionary Computation, vol. 3, no. 4, pp. 257-271. 